\title{
Rice OsRH58, a chloroplast DEAD-box RNA helicase, improves salt or drought stress tolerance in Arabidopsis by affecting chloroplast translation
}

\author{
Ghazala Nawaz ${ }^{1,2}$ and Hunseung Kang ${ }^{1 *}$ (D)
}

\begin{abstract}
Background: Despite increasing characterization of DEAD-box RNA helicases (RHs) in chloroplast gene expression regulation at posttranscriptional levels in plants, their functional roles in growth responses of crops, including rice (Oryza sativa), to abiotic stresses are yet to be characterized. In this study, rice OsRH58 (LOC_Os01g73900), a chloroplast-localized DEAD-box $\mathrm{RH}$, was characterized for its expression patterns upon stress treatment and its functional roles using transgenic Arabidopsis plants under normal and abiotic stress conditions.

Results: Chloroplast localization of OsRH58 was confirmed by analyzing the expression of OsRH58-GFP fusion proteins in tobacco leaves. Expression of OsRH58 in rice was up-regulated by salt, drought, or heat stress, whereas its expression was decreased by cold, UV, or ABA treatment. The OsRH58-expressing Arabidopsis plants were taller and had more seeds than the wild type under favorable conditions. The transgenic plants displayed faster seed germination, better seedling growth, and a higher survival rate than the wild type under high salt or drought stress. Importantly, levels of several chloroplast proteins were increased in the transgenic plants under salt or dehydration stress. Notably, OsRH58 harbored RNA chaperone activity.

Conclusions: These findings suggest that the chloroplast-transported OsRH58 possessing RNA chaperone activity confers stress tolerance by increasing translation of chloroplast mRNAs.
\end{abstract}

Keywords: Abiotic stress, Arabidopsis, Chloroplast, DEAD-box RH, Rice, RNA helicase

\section{Background}

Plants are sessile organisms facing diverse environmental stresses, such as temperature shock, high salinity, drought, and UV. These stresses severely affect plant growth and productivity. Photosynthesis in chloroplasts is an essential cellular process that is greatly affected by environmental stresses $[1,2]$. To withstand environmental stresses, plants need to regulate cellular metabolic processes, including photosynthesis, that are crucial for plant growth and productivity under abiotic stresses $[3,4]$. Chloroplast gene expression is regulated at the level of pre-RNA processing, base editing, splicing, decay, and translation

\footnotetext{
* Correspondence: hskang@chonnam.ac.kr

${ }^{1}$ Department of Applied Biology, College of Agriculture and Life Sciences,

Chonnam National University, 77 Yongbong-ro, Buk-gu, Gwangju 61186,

South Korea

Full list of author information is available at the end of the article
}

[5-7]. Although fewer than 150 proteins are encoded by the chloroplast genome, the chloroplast contains more than 3000 proteins that are encoded by the nucleus genome and transported into the chloroplast. Therefore, inter-communications between the chloroplast and the nucleus via bidirectional signaling are required for fine-tuned gene expression in both organelles [8-13].

Many RNA-binding proteins (RBPs), including DEADbox RNA helicases (RHs), are transported to the chloroplast. RNA helicases are proteins that can unwind RNAs and remodel structured RNAs [14, 15]. Many previous reports have demonstrated that DEAD-box RHs are cellular molecules important for plant growth and fitness in harsh environments (reviewed in [16, 17]). Although many DEAD-box RHs in the nucleus have been characterized in

(c) The Author(s). 2019 Open Access This article is distributed under the terms of the Creative Commons Attribution 4.0 International License (http://creativecommons.org/licenses/by/4.0/), which permits unrestricted use, distribution, and reproduction in any medium, provided you give appropriate credit to the original author(s) and the source, provide a link to the Creative Commons license, and indicate if changes were made. The Creative Commons Public Domain Dedication waiver (http://creativecommons.org/publicdomain/zero/1.0/) applies to the data made available in this article, unless otherwise stated. 
plants, particularly in Arabidopsis thaliana, functions of the many chloroplast-localized DEAD-box RHs in crops, including rice, are yet to be characterized. Chloroplast-localized AtRH3, HVD1, and BrRH22 have been shown to increase plant growth under drought or salinity stress [18-20]. Chloroplast-localized AtRH3 or mitochondria-localized ABO6 regulate auxin or ABA signaling [19, 21, 22]. A genome-wide search for the putative chloroplast DEAD-box RHs in Arabidopsis, maize (Zea mays), rice (Oryza sativa), and wheat (Triticum aestivum) has revealed that approximately 7 to 12 nucleus-encoded DEAD-box RHs contain potential chloroplast-targeting signal peptides [17].

Rice is an economically important cereal crop, the productivity of which is severely diminished by diverse abiotic stresses [23]. Given the possibility of using chloroplast-localized DEAD-box RHs to engineer stress-tolerant crops, it is important to determine the roles of chloroplast-localized DEAD-box RHs in rice under stress conditions. Despite the increasing notifications on the potential roles of chloroplast-localized DEAD-box RHs in plant fitness under abiotic stresses, the roles of DEAD-box RHs in rice chloroplasts are largely unknown. To date, only a few RHs, such as OsABP and OsRH53, have been characterized in rice's response to abiotic stresses [24, 25]. In this study, we characterized the function of a chloroplast-localized OsRH58 (LOC_Os01g73900) in abiotic stress response by using transgenic Arabidopsis plants.

\section{Results}

Subcellular localization and stress-responsive expression patterns of OsRH58 in rice

OsRH58 was identified as a putative chloroplast-localized DEAD-box RH in a previous study [17]. It consists of 438 amino acid residues and contains a 60 amino acid-long putative chloroplast transit peptide (cTP) at the $\mathrm{N}$-terminal end (Fig. 1a). To confirm whether OsRH58 is localized to chloroplasts, the Agrobacterium containing the OsRH58-GFP vector was infiltrated into tobacco leaves, and the transiently expressed OsRH58-GFP fusion proteins were observed using a confocal microscope. GFP signals and chloroplast auto fluorescence were merged together (Fig. 1b), confirming chloroplast localization of the OsRH58 protein.

To analyze the stress-response expression patterns of OsRH58 in rice, different abiotic stresses were applied to rice seedlings, and OsRH58 levels were measured by quantitative RT-PCR analysis. The degree and adequacy of stress treatment in rice was shown in the previous report [25]. Drought, salt, or heat stress increased the expression of $O s R H 58$ up to two-fold. Cold stress or ABA marginally decreased OsRH58 levels, whereas UV stress significantly decreased the expression of OsRH58 (Fig. 2).

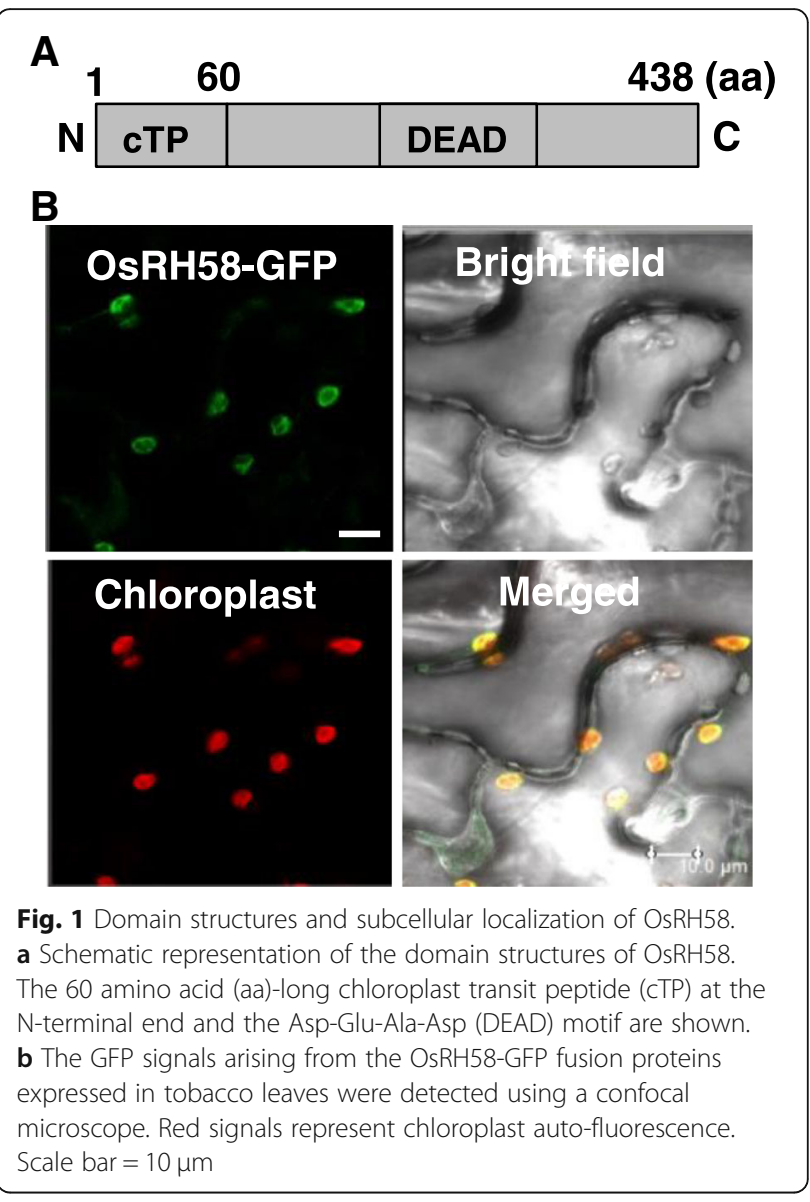

\section{OsRH58 increases the growth and seed yield of Arabidopsis plants under normal conditions}

Given that abiotic stresses modulate OsRH58 expression, it is interesting to determine the functional roles of OsRH58 in stress responses. Although we need to analyze the knockout mutant of OsRH58 to fully understand its function in rice, in this study we evaluated the function of OsRH58 using transgenic Arabidopsis plants that express OsRH58 because the OsRH58 mutant was not available. The expression of OsRH58 in the three independent homozygous lines, which displayed similar phenotypes, was confirmed by RT-PCR (Additional file 1 a). The wild type and transgenic Arabidopsis seeds germinated equally well without differences on normal MS medium (Additional file $1 \mathrm{~b}$ ). However, the transgenic plants had slightly longer roots than did the wild type (Fig. 3a). At maturity, the transgenic Arabidopsis plants were taller and had more seeds than the wild type (Fig. $3 \mathrm{~b}$ and $\mathrm{c}$ ), and the maximum quantum yields of PSII $(\mathrm{Fv} / \mathrm{Fm})$ of the wild type and transgenic Arabidopsis plants were approximately 0.82 and 0.9 , respectively (Fig. 3d). These results indicate that OsRH58 promotes plant growth and seed yield under normal conditions. 


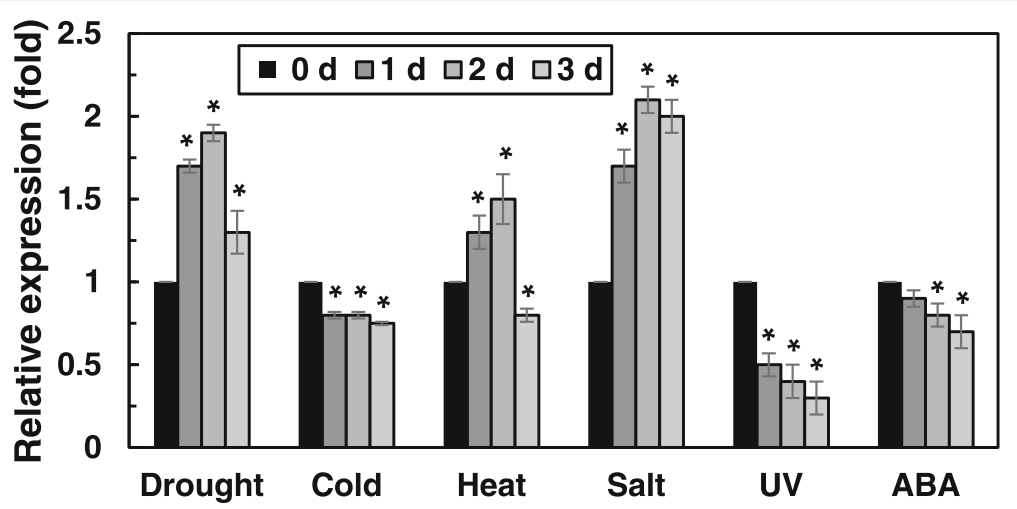

Fig. 2 Expression of OsRH58 in rice under abiotic stress treatment. Three-week-old rice seedlings were subjected to drought, $100 \mu \mathrm{M}$ ABA, cold $\left(10^{\circ} \mathrm{C}\right)$, UV $\left(5\right.$ watt), salt $(150 \mathrm{mM} \mathrm{NaCl})$, or heat $\left(45^{\circ} \mathrm{C}\right)$, and OsRH58 levels were measured by qRT-PCR. The mean and standard error were obtained from three biological replicates, and asterisks indicate statistically significant differences $(P \leq 0.05)$

OsRH58 has positive effects on seed germination of Arabidopsis under salinity or drought stress

The role of OsRH58 in seed germination under stress was evaluated by comparing germination rates of the wild type and transgenic seeds under various abiotic stress treatments. Approximately 50 and $85 \%$ of transgenic seeds germinated on the second and third day, respectively, on MS medium containing $150 \mathrm{mM} \mathrm{NaCl}$, whereas approximately
25 and $65 \%$ of wild type seeds germinated on the same days (Fig. 4a). Given $300 \mathrm{mM}$ mannitol treatment, approximately 25 and $80 \%$ of transgenic seeds germinated on the second and third day, respectively, whereas only 3 and $40 \%$ of wild type seeds germinated on the same days (Fig. 4a). In contrast, the wild type and transgenic seeds germinated without noticeable differences under cold stress or ABA treatment (Additional file $1 \mathrm{~b}$ ).

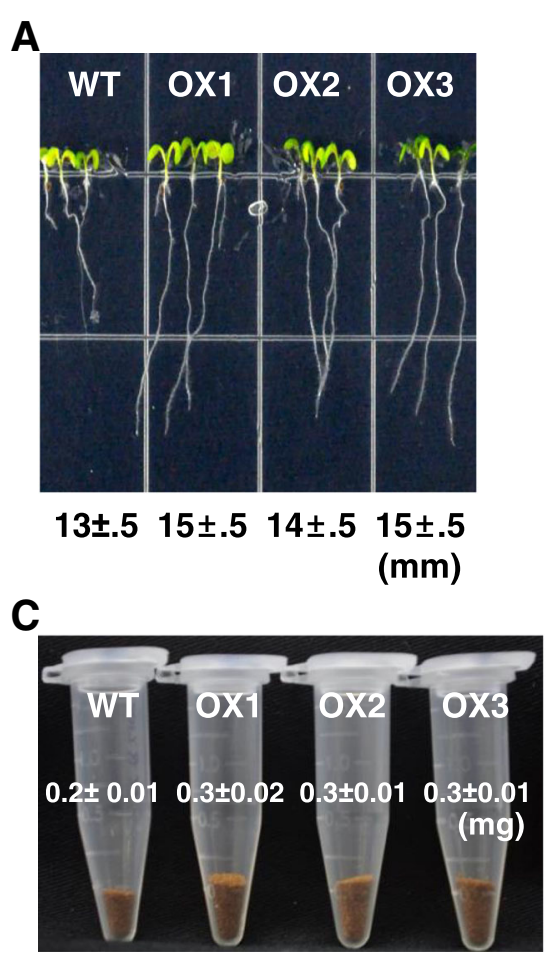

\section{B}

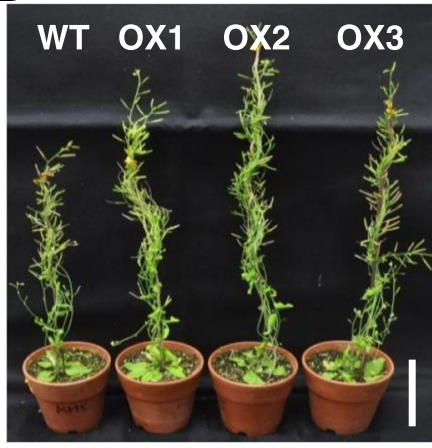

$280 \pm 7330 \pm 5350 \pm 5330 \pm 5$

(cm)

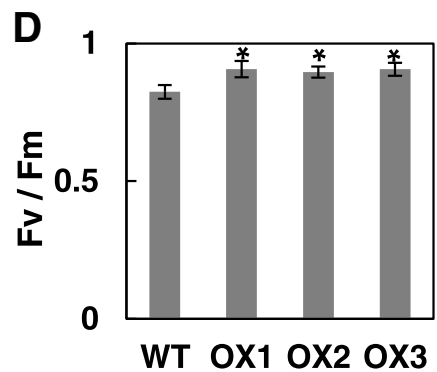

Fig. 3 OsRH58 improves Arabidopsis growth under normal conditions. a Root length of the wild type (WT) and OsRH58-expressing transgenic Arabidopsis plants (OX1, OX2, and OX3) was measured on MS medium 14 days after germination. b Plant height and $\mathbf{c}$ seed yield were measured at maturity. $\mathbf{d}$ The maximum quantum yield ( $\mathrm{Fv} / \mathrm{Fm}$ ) of PSII was measured 4 weeks after germination. The mean and standard error were obtained from three biological replicates, and asterisks indicate statistically significant differences $(P \leq 0.05)$ 


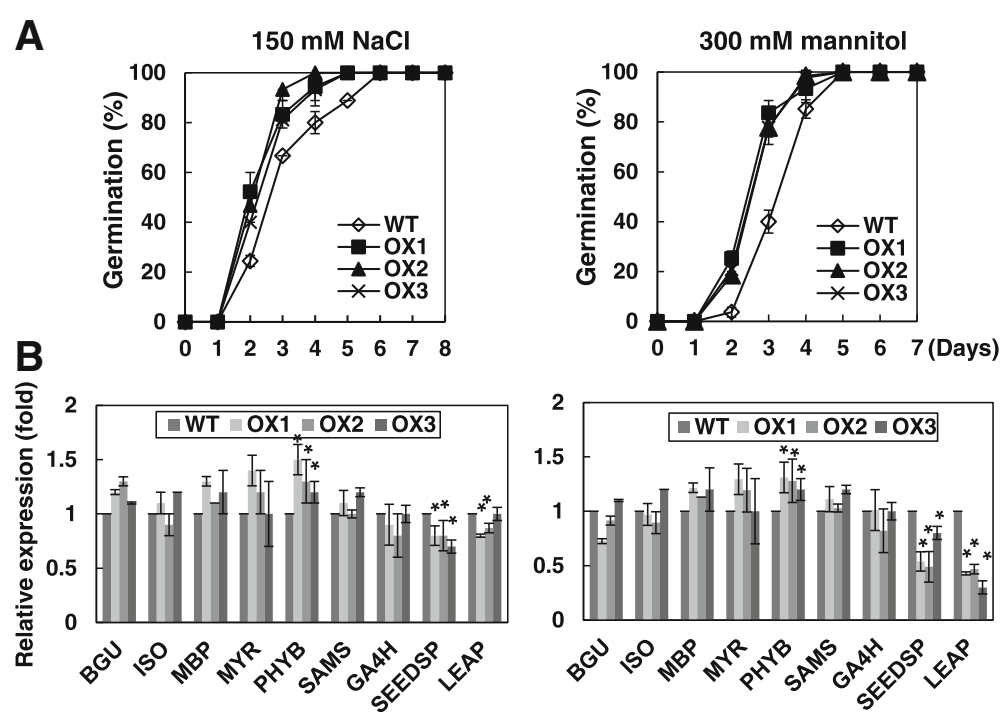

Fig. 4 OsRH58 accelerates seed germination under salt or dehydration stress. a Germination rates of the wild type (WT) and OsRH58-expressing transgenic Arabidopsis plants (OX1, OX2, and OX3) were scored on MS medium supplemented with $150 \mathrm{mM} \mathrm{NaCl}$ or $300 \mathrm{mM} \mathrm{Mannitol.}$ b Transcript levels of each gene were measured at the second day by qRT-PC. The mean and standard error were obtained from three biological replicates, and asterisks indicate statistically significant differences $(P \leq 0.05)$

To understand how OsRH58 affects seed germination under high salt or dehydration stress, we examined expression levels of germination-responsive genes, including the positive effectors of germination, such as $\beta$-glucosidase (BGU), GA4 homolog (GA4H), isocitrate lyase (ISO), myrosinase (MYR), myrosinase-binding protein (MBP), phytochrome B (PHYB), and S-adenosyl methionine synthetase (SAM) $[26,27]$, and the negative effectors of seed germination, such as seed storage protein (SEEDSP) and LEA protein (LEAP) [27]. Results showed that the level of a positive effector $P H Y B$ was marginally increased in transgenic plants under salinity or dehydration stress, whereas the transgenic Arabidopsis plants had much lower levels of negative effectors (SEEDSP and LEAP) than the wild type (Fig. 4b). These results suggest that the better germination of the transgenic seeds under salinity or dehydration stress partly results from the modulation of these effectors of germination by OsRH58.

\section{OsRH58-expressing transgenic plants are tolerant to salinity or drought stress}

When the wild type and OsRH58-expressing Arabidopsis plants were grown under normal, cold, or ABA conditions, their seedling growth was similar to each other (Additional file 2). In contrast, the transgenic plants tolerated salinity or drought stress much better. The transgenic Arabidopsis plantlets showed marginally better seedling growth and noticeably better root growth than the wild type on MS medium containing $150 \mathrm{mM} \mathrm{NaCl}$ (Fig. 5). When subjected to dehydration stress on MS medium with $300 \mathrm{mM}$ mannitol, the fresh weight and root length of the OsRH58-expressing transgenic Arabidopsis plants were significantly higher than those of the wild type (Fig. $6 \mathrm{a}$ and b). To further evaluate the role of OsRH58 in drought stress tolerance, survival rates of the wild type and OsRH58-expressing transgenic seedlings grown in soil were measured after recovering from four days of drought stress treatment. Approximately $85 \%$ of the transgenic seedlings survived after recovery, whereas approximately $50 \%$ of the wild type seedlings survived (Fig. 6c). These results indicate that OsRH58 confers salt or drought tolerance but not cold tolerance.

\section{OsRH58 increases chloroplast protein levels under salt or dehydration stress}

The next important question is how OsRH58 confers stress tolerance. Because OsRH58 is localized in chloroplasts, it should be involved in chloroplast RNA metabolism. We, therefore, first analyzed the efficiency of the splicing of chloroplast introns under normal, salt, or dehydration stress conditions. Notably, the splicing efficiencies of chloroplast introns in the wild type and OsRH58-expressing transgenic Arabidopsis plants were comparable to each other under normal, salinity, or dehydration conditions (Additional file 3). We next assessed by immunoblot analysis whether OsRH58 affects translation of chloroplast transcripts. In this study, the levels of casein lytic proteinase B3 (ClpB3), D1 protein of PSII (PsbA), cytochrome f protein (PetA), protochlorophilide oxidoreductase (POR), rubisco large subunit (RbcL), and beta subunit of ATP synthase 
$150 \mathrm{mM} \mathrm{NaCl}$

A
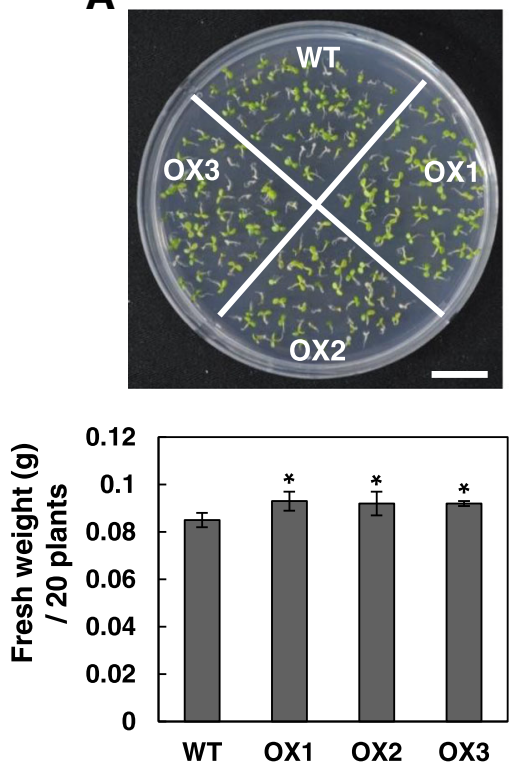

B
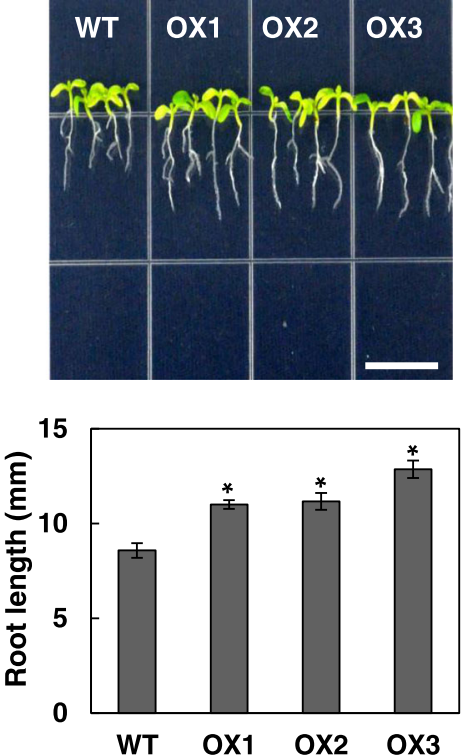

Fig. 5 OsRH58-expressing transgenic plants are tolerant against salinity stress. The wild type (WT) and OsRH58-expressing transgenic Arabidopsis plants (OX1, OX2, and OX3) were grown on MS medium containing $150 \mathrm{mM} \mathrm{NaCl}$, and (a) seedling fresh weight and (b) root length were measured after 3 weeks and 2 weeks, respectively. The mean and standard error were obtained from three biological replicates, and asterisks indicate statistically significant differences $(P \leq 0.05)$. Scale bar $=1 \mathrm{~cm}$

(AtpB) were examined, due to the availability of these antibodies in our laboratory. Equal loading of total proteins in each lane on the gel was verified by Ponceau-S staining (Additional file 4). The results showed that the OsRH58-expressing transgenic plants accumulated much larger amounts of ClpB3, PsbA, PetA, POR, and RbcL than did the wild type under salt or dehydration stress (Fig. 7). In comparison, the wild type and transgenic plants had similar amounts of proteins when tested under normal conditions (Fig. 7). Altogether, these data suggest that OsRH58 confers stress tolerance by influencing the translation of chloroplast mRNAs.

\section{OsRH58 harbors RNA chaperon activity}

The next important question is how OsRH58 affects translation of chloroplast genes. Considering that many DEAD-box RHs participate in translation control by functioning as RNA chaperones [28-30], it is possible that OsRH58 participates in the translation of chloroplast mRNAs through RNA chaperone activity. We tested this possibility by analyzing the RNA chaperone activity of OsRH58. We, first, evaluated the complementation capability of OsRH58 in E. coli mutant BX04 cell that is sensitive to cold shock because of the lack of RNA chaperones [31]. The BX04 cells harboring OsRH58, CspA (used as a positive control), or pINIII (used as a negative control) grew similarly at a normal temperature $\left(37^{\circ} \mathrm{C}\right)$. In contrast, the BX04 cells harboring OsRH58 or CspA grew well at a low temperature $\left(20^{\circ} \mathrm{C}\right)$, whereas the growth of the pINIII-harboring BX04 cells was poor at $20^{\circ} \mathrm{C}$ (Fig. 8a). We next evaluated the base pairs-breaking ability of OsRH58 in E. coli RL211 cells in which the chloramphenicol $(\mathrm{Cm})$-resistant gene is expressed if base pairs on the RNA are disrupted in trpL terminator located upstream of the Cm-resistant gene [32]. The RL211 cells having either OsRH58 or CspA grew well on LB medium containing chloramphenicol, whereas the RL211cells possessing pINIII did not grow (Fig. 8). These results confirm that OsRH58 harbors RNA chaperone activity.

\section{Discussion}

Despite the potential role of chloroplast-localized DEAD-box RHs in plant adaptation to abiotic stresses, reports characterizing the chloroplast localization and function of DEAD-box RHs in crop species are few. The current results show that the chloroplast-localized rice OsRH58 can improve seed germination, seedling growth, and plant vigor under both normal and stress conditions. Notably, OsRH58 increased seed yield in Arabidopsis under normal conditions (Fig. 3). It would be valuable to further evaluate whether OsRH58 can be used to increase seed yield in rice under normal growth conditions. Constitutive expression of OsRH58 in Arabidopsis accelerated seed germination and increased seedling growth and survival when subjected to salt or dehydration stress (Figs. 4, 5, and 6). Because the expression of 


\section{$300 \mathrm{mM}$ mannitol}

A
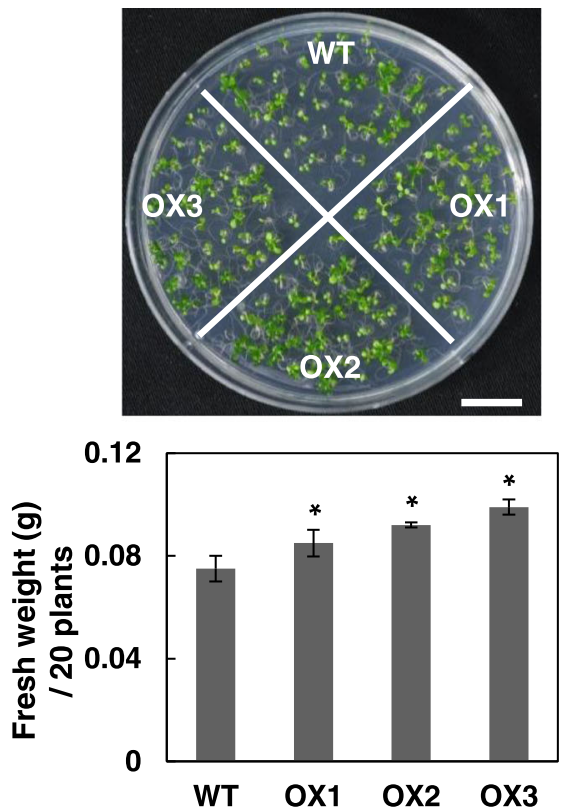

C Before drought

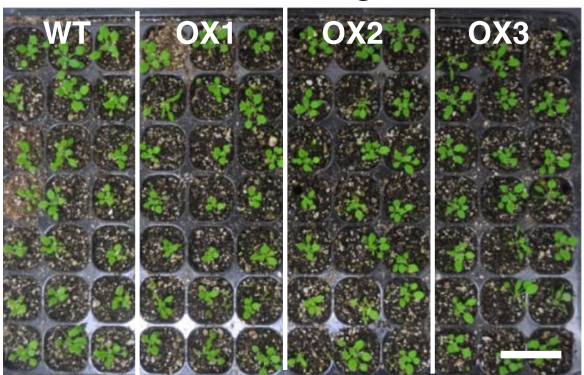

B
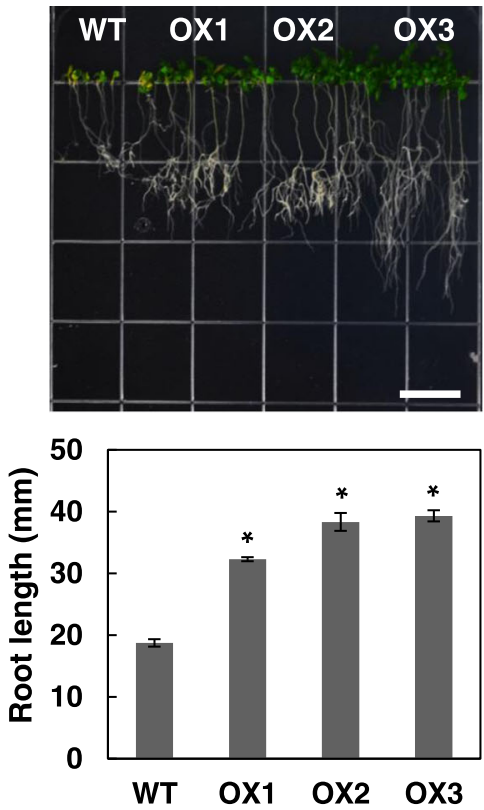

Fig. 6 OsRH58-expressing transgenic plants are tolerant against drought stress. The wild type (WT) and OsRH58-expressing transgenic Arabidopsis plants (OX1, OX2, and OX3) were grown on MS medium containing $300 \mathrm{mM}$ mannitol, and (a) seedling fresh weight and (b) root length were measured after 3 weeks and 2 weeks, respectively. c Two-week-old seedlings in soil were unwatered for 4 days, and survival rates were scored at 1 week after recovery. The mean and standard error were obtained from three biological replicates, and asterisks indicate statistically significant differences $(P \leq 0.05)$. Scale bar $=1 \mathrm{~cm}$

OsRH58 increased in rice under salt or drought stress (Fig. 2), it is likely that OsRH58 performs a similar positive role in rice under salt or drought stress. It would be valuable to evaluate whether OsRH58 can be used to engineer salt- or drought-tolerant rice cultivars. A recent study has also demonstrated that expression of $\mathrm{BrRH} 22$, a chloroplast-transported DEAD-box RH in cabbage (Brassica rapa), whose transcript level is markedly up-regulated by salt, drought, cold, or heat stress, improves germination and plant vigor in transgenic Arabidopsis under salt or drought stress [25]. In contrast, expression of nuclear OsRH53, whose transcript level was decreased in rice by salt or drought stress, had a negative effect on the germination and growth of Arabidopsis under salt or dehydration stress [25]. These results suggest that expression patterns of DEAD-box RHs in crops modulated by abiotic stresses are closely correlated with their stress-tolerance functions. However, it was also noted that, although the expression of rice OsRH58 and cabbage BrRH22 was increased by cold stress, the OsRH58- or BrRH22-expressing transgenic Arabidopsis showed no tolerance to cold stress (20; Additional file 2). These results suggest that more functional analysis of DEAD-box RHs is required in crop species to fully understand their roles in stress tolerance. Moreover, recent systematic analyses of the organelle-targeted or potential DEAD-box $\mathrm{RHs}$ in crops such as rice, maize, wheat, and tomato highlighted the number and proposed roles of DEAD-box 


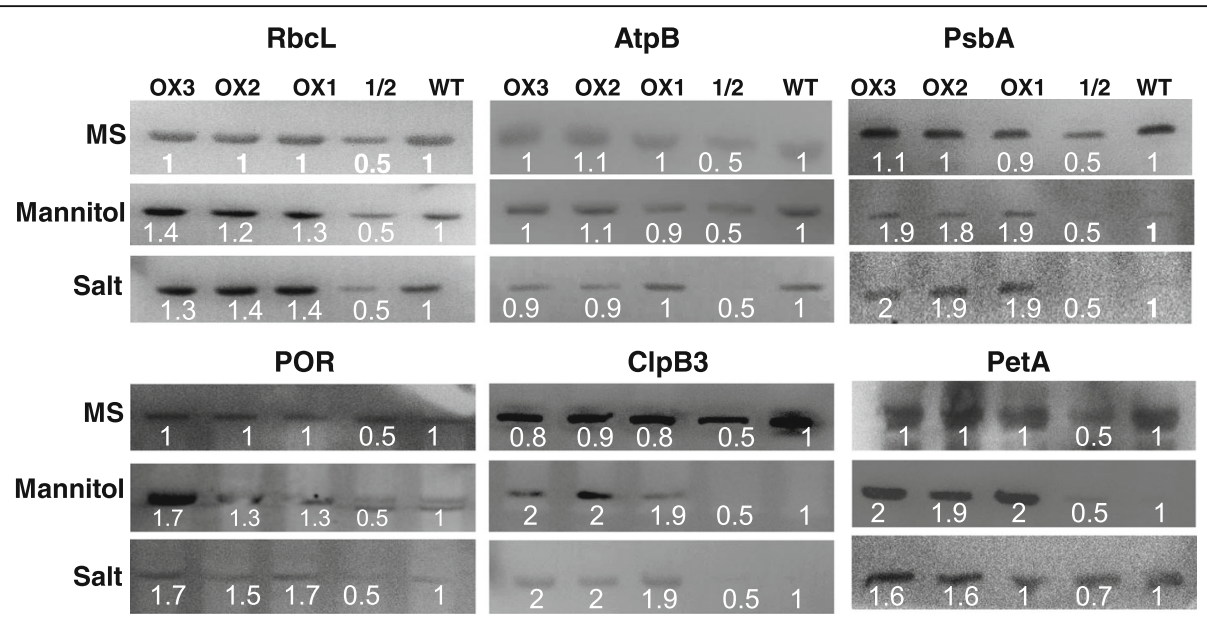

Fig. 7 OsRH58 affects the levels of chloroplast proteins under salt or dehydration stress. Total proteins were extracted from 2-week-old wild type (WT) and OsRH58-expressing transgenic Arabidopsis plants (OX1, OX2, and OX3) grown on MS medium containing $300 \mathrm{mM}$ mannitol or $150 \mathrm{mM}$ $\mathrm{NaCl}$. Twenty to thirty micrograms of total proteins were separated on SDS-12\% PAGE, and the proteins were detected with the antibody raised against each protein. The intensities of each band were calculated, and the mean and standard error were obtained from three biological replicates. 1/2, half amount of WT protein

RHs in growth, development, and stress responses in crops [17, 33]. The functions and significance of these DEAD-box RHs in crops await further characterization.

It is important to understand the cellular roles of chloroplast DEAD-box RHs. Considering that RNA metabolic processes, including intron splicing, pre-RNA processing, editing, and translation, are main regulatory steps in chloroplast gene expression [5, 7], DEAD-box RHs should be involved in these cellular processes. Previous

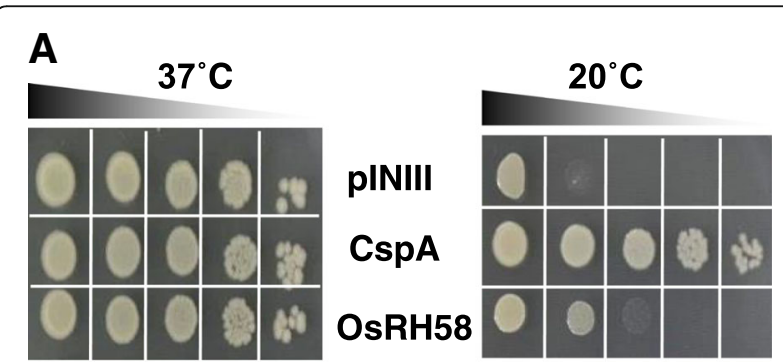

B

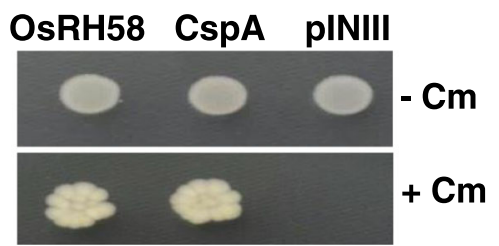

Fig. 8 OsRH58 possesses RNA chaperone activity. a The complementation ability of OsRH58 in Escherichia coli BX04 mutant cells was evaluated by incubating the diluted cell cultures at $20^{\circ} \mathrm{C}$. $\mathbf{b}$ The transcription anti-termination activity of OsRH58 in E. coli RL211 mutant cells was evaluated by growing the cells on LB agar medium with (+) or without (-) chloramphenicol. The pINIII vector and pINIIICspA were used as negative and positive control, respectively studies clearly demonstrated that the chloroplast-localized DEAD-box RHs, ISE2 and RH3, are involved in the splicing of chloroplast introns in Arabidopsis [19, $21,34]$. A recent overview emphasizes the roles of DEAD-box RHs in rRNA processing during plant growth and stress responses [35]. Our current results showed that OsRH58 has no role in the splicing of chloroplast introns (Additional file 3) but influences translation of several chloroplast transcripts (Fig. 7). Several RHs have been known to participate in translation control [36]. The chloroplast-localized Arabidopsis DEAD-box AtRH22 and its cabbage ortholog BrRH22 are involved in the assembly of $50 \mathrm{~S}$ ribosomes and translation of chloroplast transcripts [20, 37]. In addition, the chloroplast-targeted ISE2 and RH50 play essential roles in chloroplast rRNA maturation in Arabidopsis [38, 39]. These current and previous findings altogether suggest the crucial role of DEAD-box RHs in translation of chloroplast mRNAs. Notably, alteration in chloroplast translation is an adaptive response of plants to abiotic stress [40]. Further studies are required to fully understand how OsRH58 influences translation of chloroplast mRNA under salt or drought stress.

Discovering the mechanistic role of OsRH58 in chloroplast translation is a remaining critical question. Because RNA molecules are prone to adapt alternative non-functional structures under stress, resolving these misfolded structures are essential for proper function of RNAs. RNA chaperones are RBPs that can rearrange the structure of diverse RNA molecules [41-43]. The importance of RHs as RNA chaperones in plant stress responses has been emerging (reviewed in [29, 30, 44, 45]). Notably, the bacterial and yeast DEAD-box RHs 
act as RNA chaperones in the process of intron splicing [46-50]. The Arabidopsis RH3 participates in the splicing of chloroplast introns via its RNA chaperone activity [19]. Our findings show that OsRH58 possesses RNA chaperone activity (Fig. 8), which influences the translation of several chloroplast mRNAs. Importantly, RNA chaperones are known to bind to RNA targets with a low sequence preference [28]. It is likely that OsRH58 binds to diverse chloroplast mRNAs, and its RNA chaperone activity rearranges the RNA structures, which affects the translation of several target mRNAs such as POR, RbcL, ClpB3, PsbA, and PetA (Fig. 7). Because OsRH58 does not affect the levels of chloroplast proteins under normal conditions (Fig. 7), further studies are required to find out whether the structures of chloroplast mRNAs change in ways that depend on specific stress conditions and to fully understand how OsRH58 influences translation of chloroplast mRNA under salt or drought stress.

\section{Conclusions}

Our results highlight the importance of chloroplast-localized OsRH58 in plant growth and vigor under abiotic stresses. Although it is clear that OsRH58 helps to improve the germination and growth of Arabidopsis under salt or drought stress, the stress-responsive role of OsRH58 in rice remains to be investigated. In particular, it would be interesting to determine whether chloroplast-located OsRH58 affects the expression or activity of ROS scavenging enzymes that are deeply associated with stress tolerance in plants. Furthermore, evaluation of the functions of as yet uncharacterized chloroplast-transported DEAD-box RHs and their cellular roles in stress response awaits further investigation. Considering the potential applications of DEAD-box RHs in developing stress-tolerant crops, it would be of value to engineer DEAD-box RHs using a CRISPR-Cas9 genome editing technology to introduce or delete specific DEAD-box RHs whose expression are up- or down-regulated by abiotic stresses.

\section{Methods}

\section{Determination of subcellular localization of OsRH58}

OsRH58 was identified in a previous study as a putative chloroplast-localized DEAD-box RH that contains a potential chloroplast transit peptide (cTP) at the N-terminal end [17]. To confirm the chloroplast localization of OsRH58, Agrobacterium tumefaciens GV3101 harboring the CsV-OsRH58-GFP vector was infiltrated into tobacco leaves, and the GFP signals were examined using a laser scanning confocal microscope (Carl Zeiss Inc., Thornwood, NY, USA) with the excitation wavelength of $488 \mathrm{~nm}$ and the emission wavelength of 505-545 nm. Chloroplast auto-fluorescence was examined with the excitation and emission wavelengths of $633 \mathrm{~nm}$ and 640-690 nm, respectively.

\section{Rice materials and stress treatment}

The $A$. thaliana used in this study was Columbia- 0 ecotype, which was obtained from the Arabidopsis Biological Resource Center (Columbus, OH, USA). Rice (Dongjin variety) seeds were obtained from the Crop Biotech Institute of Kyung Hee University (Suwon, Korea). Rice seeds were sown on Murashige and Skoog (MS) medium, and germinated seedlings were transferred to soil and kept growing in a growth room at $30^{\circ} \mathrm{C}$ under $16 / 8 \mathrm{~h}$ light/dark cycles. Various abiotic stresses such as drought, salt, cold, or UV, and ABA were applied to four-week-old rice plantlets as previously described [25]. Briefly, watering was stopped for drought stress, and the pots were immersed in $300 \mathrm{mM} \mathrm{NaCl}$ solution for salt stress. Cold or heat stress was applied by growing the plants at $10^{\circ} \mathrm{C}$ or $39^{\circ} \mathrm{C}$, respectively. The rice seedlings were grown under $3.0 \mathrm{~W}$ $\mathrm{m}^{-2}$ UV light for UV stress, and $100 \mu \mathrm{M}$ ABA solution was sprayed onto the plants.

\section{RNA extraction and real-time RT-PCR analysis}

Plant materials were ground, and total RNA was extracted using a Plant RNeasy kit (Qiagen, Valencia, CA, USA). Real-time quantification of transcript levels was performed on a Rotor-Gene Q (Qiagen) using MG2X SYBR Green RT-PCR kit (Qiagen) as described [51]. Splicing efficiency was measured by qRT-PCR with primers to amplify exon/exon (spliced or mature) and intron/exon (unspliced or precursor) transcripts as described [52, 53]. Primers used in qRT-PCR are shown in Additional file 5. Action was used as a reference gene.

\section{Generation of OsRH58-expressing transgenic Arabidopsis plants}

The cDNA encoding OsRH58 was amplified by RT-PCR and cloned into pCAMBIA1301 vector, which directs constitutive expression of OsRH58 under the cauliflower mosaic virus $35 \mathrm{~S}$ promoter. Vacuum infiltration [54] using Agrobacterium tumefaciens GV3101 was used to transform Arabidopsis. Selection of transgenic Arabidopsis plants was carried out on MS medium supplemented with hygromycin $\left(50 \mu \mathrm{g} \mathrm{mL}^{-1}\right)$ and carbenicillin $(250 \mu \mathrm{g}$ $\left.\mathrm{mL}^{-1}\right)$. Homozygous $\mathrm{T}_{4}$ lines were obtained and used for subsequent analysis. To confirm the expression of OsRH58 in transgenic Arabidopsis plants, RT-PCR was performed using the primers listed in Additional file 5.

\section{Seed germination and seedling growth assays under abiotic stresses}

The wild type and transgenic seeds were sown on MS medium containing $1 \%$ sucrose. The plates were kept at $4{ }^{\circ} \mathrm{C}$ in the dark for three days for stratification and then 
were incubated at $25^{\circ} \mathrm{C}$ under $16 / 8 \mathrm{~h}$ light/dark cycles. The effects of high salt or drought stress on seed germination were evaluated by sowing the seeds on MS medium containing either $150-200 \mathrm{mM} \mathrm{NaCl}$ or $300-$ $450 \mathrm{mM}$ mannitol, respectively. The effects of cold stress on seed germination were evaluated by incubating the plates at $10^{\circ} \mathrm{C}$, and $\mathrm{ABA}$ effects on seed germination were analyzed by sowing the seeds on MS medium with 1-5 $\mu \mathrm{M}$ ABA. Germination rate was scored by observing radicle protrusion. To evaluate the effects of different stresses on root and seedling growth, 3-day-old seedlings that were fully germinated on normal MS medium were transferred to MS plates supplemented with $\mathrm{NaCl}$ or mannitol, or incubated at low temperatures as described above. To measure root length, the MS plates were incubated vertically.

\section{Measurement of photosynthetic activity}

Leaves of the wild type and transgenic plants were adapted to darkness for $20 \mathrm{~min}$, and the maximum quantum yield of PSII ( Fv/Fm) was measured using Handy PEA chlorophyll fluorimeter (Hansatech instruments Ltd., England) according to the manufacturer's instruction.

\section{Protein extraction and immunoblot analysis}

Plant materials were ground, and total proteins were extracted in a buffer $(50 \mathrm{mM}$ Tris- $\mathrm{HCl}, 100 \mathrm{mM}$ DTT, pH 7.5, $1 \mathrm{mM}$ EDTA, $250 \mathrm{mM}$ sucrose, $5 \mathrm{mM}$ leupeptin and $100 \mathrm{mM}$ PMSF). A SDS-12\% polyacrylamide gel electrophoresis was used to separate proteins, after which proteins on the gel were blotted to a polyvinylidene difluoride membrane. The primary antibody specific to each protein was incubated with the membrane. After binding the secondary antibody with horse radish peroxidase, chemiluminescence signals were detected with an image analyzer (GE Healthcare Life Sciences, USA). Ponceau-S solution containing $5 \%$ acetic acid and $0.1 \%$ Ponceau-S was used to stain the total proteins on the membrane.

\section{RNA chaperone activity assay}

The cDNA encoding OsRH58 was amplified by RT-PCR and cloned into a pINIII vector. For a complementation assay in Escherichia coli BX04 mutant cells [31], the pINIII-OsRH58, pINIII-CspA, or pINIII vector was introduced into the cell. Serially diluted cultures of BX04 cells expressing each construct were spotted on Luria-Bertani (LB) agar plates, and the plates were incubated at $20^{\circ} \mathrm{C}$. Transcription anti-termination assay was performed by using the E. coli RL211 cells [32]. The pINIII-OsRH58, pINIII-CspA, or pINIII vector was introduced into the RL211 cells, and cultures of the transformants were spotted on LB plates containing tetracycline and with or without chloramphenicol $(\mathrm{Cm})$. The plates were incubated at $37^{\circ} \mathrm{C}$.

\section{Statistical analysis}

The differences in gene expression levels and growth parameters between the wild type and transgenic plants were compared by $t$ test $(p \leq 0.05)$ using the SigmaPlot 10 program (Systat Software, Inc., San Jose, CA, USA).

\section{Additional files}

\begin{abstract}
Additional file 1: Confirmation and seed germination of transgenic plants under normal, cold, or ABA conditions. (A) Expression of OsRH58 in three homozygous Arabidopsis lines (OX1, OX2, and OX3) was confirmed by RT-PCR. Actin was used as a loading control. (B) Germination rates of the wild type (WT) and transgenic plants were scored on MS medium or MS medium supplemented with $1 \mu \mathrm{M}$ ABA at normal temperatures, and on MS medium at $10^{\circ} \mathrm{C}$. (PDF $406 \mathrm{~kb}$ )
\end{abstract}

Additional file 2: Seedling growth of the wild type and transgenic plants under normal, cold, or ABA conditions. Growth of the wild type (WT) and OsRH58-expressing transgenic Arabidopsis plants (OX1,OX2, and OX3) was analyzed on MS medium or MS medium supplemented with $2 \mu \mathrm{M}$ ABA at normal temperatures, and on MS medium at $10^{\circ} \mathrm{C}$. The mean and standard error of fresh weight were obtained from three biological replicates. (PDF $408 \mathrm{~kb}$ )

Additional file 3: Splicing efficiency of chloroplast intron-containing genes. Total RNA was extracted from 2-week-old wild type (WT) and OsRH58-expressing transgenic Arabidopsis plants (OX1,OX2, and OX3) grown on MS medium or MS medium supplemented with $150 \mathrm{mM} \mathrm{NaCl}$ or 300 $\mathrm{mM}$ mannitol, and the levels of unspliced and spliced transcripts of each gene were determined by real-time RT-PCR. The mean and standard error were obtained from three biological replicates. (PDF $405 \mathrm{~kb}$ )

Additional file 4: SDS-PAGE gels showing total proteins in each sample. Total proteins were extracted from 2-week-old wild type (WT) and OsRH58-expressing transgenic Arabidopsis plants (OX1,OX2, and OX3) grown on MS medium or MS medium supplemented with $150 \mathrm{mM} \mathrm{NaCl}$ or $300 \mathrm{mM}$ mannitol, and the proteins were separated on SDS-12\% PAGE gel, transferred to membrane, and stained with a Ponceau-S. 1/2, half amount of WT protein. (PDF $408 \mathrm{~kb}$ )

Additional file 5: List of primers used in RT-PCR and quantitate realtime RT-PCR analysis. (PDF $403 \mathrm{~kb}$ )

\section{Abbreviations}

DEAD: asp-glu-ala-asp; RBP: RNA-binding protein; $\mathrm{RH}$ : RNA helicase

\section{Acknowledgements}

We thank Drs. M. Inouye and S. Phadtare for the BX04 mutant cells and the pINIII vector and Dr. R. Landick for the E. coli RL211 cells.

\section{Funding}

This work was supported by grants from the Next-Generation BioGreen21 Program (PJ01314701; PJ01312201), funded by the Rural Development Administration, Republic of Korea, and by a grant from the Mid-career Researcher Program through the National Research Foundation of Korea funded by the Ministry of Science, ICT and Future Planning (NRF-

2016R1A2B4009172), Republic of Korea.

\section{Availability of data and materials}

All data can be found within the manuscript and additional files. The datasets used and/or analyzed during the current study are available from the corresponding author on reasonable request.

\section{Authors' contributions}

HK designed the experiments; GN conducted most of research and analyzed the data together with HK; GN and HK contributed to the writing of the manuscript. Both authors read and approved the final manuscript.

Ethics approval and consent to participate Not applicable 


\section{Consent for publication}

Not applicable

\section{Competing interests}

The authors declare that they have no competing interests.

\section{Publisher's Note}

Springer Nature remains neutral with regard to jurisdictional claims in published maps and institutional affiliations.

\section{Author details}

${ }^{1}$ Department of Applied Biology, College of Agriculture and Life Sciences, Chonnam National University, 77 Yongbong-ro, Buk-gu, Gwangju 61186, South Korea. ${ }^{2}$ Department of Botany, Kohat University of Science and Technology, Indus Highway Kohat, Kohat, Khyber Pakhtunkhwa 26000, Pakistan

\section{Received: 14 November 2018 Accepted: 28 December 2018}

\section{Published online: 09 January 2019}

\section{References}

1. Biswal B, Joshi PN, Raval MK, Biswal UC. Photosynthesis, a global sensorof environmental stress in green plants: stress signaling and adaptation. Curr Sci. 2011;101:47-56.

2. Singh J, Thakur JK. Photosynthesis and abiotic stress in plants. In: Vats S, editor. Biotic and abiotic stress tolerance in plants. Singapore: Springer; 2018. p. 27-46.

3. Ashraf M, Harris PJC. Photosynthesis under stressful environments: an overview. Photosynthetica. 2013;51:163-90.

4. Gururani MA, Venkatesh J, Tran LSP. Regulation of photosynthesis during abiotic stress-induced photoinhibition. Mol Plant. 2015;8:1304-20.

5. del Campo EM. Post-transcriptional control of chloroplast gene expression. Gene Regul Syst Biol. 2009;3:31-47.

6. Jacobs J, Kuck U. Function of chloroplast RNA-binding proteins. Cell Mol Life Sci. 2011;68:737-48.

7. Jung HJ, Park SJ, Kang H. Regulation of RNA metabolism in plant development and stress responses. J Plant Biol. 2013;56:123-9.

8. Pesaresi P, Schneider A, Kleine T, Leister D. Interorganellar communication. Curr Opin Plant Biol. 2007;10:600-6.

9. Stern DB, Goldschmidt-Clermont M, Hanson MR. Chloroplast RNA metabolism. Annu Rev Plant Biol. 2010;61:125-55.

10. Singh $R$, Singh $S$, Parihar $P$, Singh VP, Prasad SM. Retrograde signalingbetween plastid and nucleus: a review. J Plant Physiol. 2015;181:5-66.

11. Chan KX, Phua SY, Crisp P, Mc Quinn R, Pogson BJ. Learning the languages of the chloroplast: retrograde signaling and beyond. Annu Rev Plant Biol. 2016;67:25-53.

12. de Souza A, Wang JZ, Dehesh K. Retrograde signals: integrators of Interorganellar communication and orchestrators of plant development. Annu Rev Plant Biol. 2017:28:85-108.

13. Hernández-Verdeja T, Strand A. Retrograde signals navigate the path to chloroplast development. Plant Physiol. 2018;176:967-76.

14. Linder P, Owttrim GW. Plant RNA helicases: linking aberrant and silencing RNA. Trends Plant Sci. 2009;14:344-52.

15. Gilman B, Tijerina P, Russell R. Distinct RNA-unwinding mechanisms of DEAD-box and DEAH-box RNA helicase proteins in remodeling structured RNAs and RNPs. Biochem Soc Trans. 2017:45:1313-21.

16. Vashisht AA, Tuteja N. Stress responsive DEAD-box helicases: a new pathway to engineer plant stress tolerance. J Photochem Photobiol. 2006:84:150-60.

17. Nawaz G, Kang H. Chloroplast- or mitochondria-targeted DEAD-box RNA helicases play essential roles in organellar RNA metabolism and abiotic stress responses. Front Plant Sci. 2017;8:871.

18. Nakamura T, Muramoto Y, Yokota S, Ueda A, Takabe T. Structural and transcriptional characterization of a salt-responsive gene encoding putative ATP-dependent helicase in barley. Plant Sci. 2004;167:63-70.

19. Gu L, Xu T, Lee K, Lee KH, Kang H. A chloroplast-localized DEAD-box RNA helicase AtRH3 is essential for intron splicing and plays an important role in the growth and stress response in Arabidopsis thaliana. Plant Physiol Biochem. 2014;82:309-18

20. Nawaz G, Lee K, Park SJ, Kim YO, Kang H. A chloroplast-targeted cabbage DEAD-box RNA helicase BrRH22 confers abiotic stress tolerance to transgenic Arabidopsis plants by affecting translation of chloroplast transcripts. Plant Physiol Biochem. 2018;127:336-42.

21. Asakura Y, Galarneau E, Watkins KP, Barkan A, van Wijk KJ. Chloroplast RH3 DEAD-box RNA helicases in Zea mays and Arabidopsis thaliana function in splicing of specific group II introns and affect chloroplast ribosome. Plant Physiol. 2012:159:961-74.

22. He J, Duan Y, Hua D, Fan G, Wang L, Liu Y. DEXH-box RNA helicasemediated mitochondrial reactive oxygen species production in Arabidopsis mediates crosstalk between abscisic acid and auxin signaling. Plant Cell. 2012;24:1815-33.

23. Grover A, Minhas D. Towards the production of abiotic stress tolerant transgenic rice plants: issues, progress and future research needs. Proc Indian Nat Sci Acad. 2000:66:13-32.

24. Macovi A, Vaid N, Tula S, Tuteja N. A new DEAD-box helicase ATP-binding protein (OsABP) from rice is responsive to abiotic stress. Plant Signal Behav. 2012;7:1138-43

25. Nawaz G, Sai TZT, Lee K, Kim YO, Kang H. Rice DEAD-box RNA helicase OsRH53 has negative impact on Arabidopsis response to abiotic stresses. Plant Growth Regul. 2018:85:153-63.

26. Yamaguchi S, Smith MW, Brown RG, Kamiya Y, Sun T. Phytochrome regulation and differential expression of gibberellin $3 \beta$-hydroxylase genes in germinating Arabidopsis seeds. Plant Cell. 1998;10:2115-26.

27. Gallardo K, Job C, Groot SP, Puype M, Demol H, Vandekerckhove J, Job D. Proteomic analysis of Arabidopsis seed germination and priming. Plant Physiol. 2001;126:835-48.

28. Rajkowitsch L, Chen D, Stampfl S, Semrad K, Waldsich C, Mayer O, Jantsch MF, Konrat R, Bläsi U, Schroeder R. RNA chaperones, RNA annealers and RNA helicases. RNA Biol. 2007:4:118-30.

29. Kang H, Park SJ, Kwak KJ. Plant RNA chaperones in stress response. Trends Plant Sci. 2013:18:100-6.

30. Lee $\mathrm{K}$, Kang $\mathrm{H}$. Emerging roles of RNA-binding proteins in plant growth, development, and stress responses. Mol Cells. 2016:39:179-85.

31. Xia $B, K e ~ H$, Inouye $M$. Acquirement of cold sensitivity by quadruple deletion of the CspA family and its suppression by PNPase S1 domain in Escherichia coli. Mol Microbiol. 2001;40:179-88.

32. Landick R, Stewart J, Lee DN. Amino acid changes in conserved regions of the beta-subunit of Escherichia coli RNA polymerase alter transcription pausing and termination. Genes Dev. 1990:4:1623-36.

33. Cai J, Meng X, Li G, Dong T, Sun J, Xu T, Li Z, Han Y, Zhu M. Identification, expression analysis, and function evaluation of 42 tomato DEAD-box RNA helicase genes in growth development and stress response. Acta Physiol Plant. 2018:40:94.

34. Carlotto N, Wirth S, Furman N, Ferreyra Solari N, Ariel F, Crespi M, Kobayashi K. The chloroplastic DEVH-box RNA helicase INCREASED SIZE EXCLUSION LIMIT 2 involved in plasmodesmata regulation is required for group II intron splicing. Plant Cell Environ. 2016;39:165-73.

35. Liu Y, Imai R. Function of plant DExD/H-box RNA helicases associated with ribosomal RNA biogenesis. Front Plant Sci. 2018;9:125.

36. Jankowsky E. RNA helicases at work: binding and rearranging. Trends Biochem Sci. 2011;36:19-29.

37. Chi W, He B, Mao J, Li Q, Ma J, Ji D, Zou M, Zhang L. The function of $\mathrm{RH} 22$, a DEAD RNA helicase, in the biogenesis of the $50 \mathrm{~S}$ ribosomal subunits of Arabidopsis chloroplasts. Plant Physiol. 2012;158:693-707.

38. Bobik K, McCray T, Ernest B, Fernandez JC, Howell KA, Lane T, Staton M, Burch-Smith TM. The chloroplast RNA helicase ISE2 is required for multiple chloroplast RNA processing steps in Arabidopsis thaliana. Plant J. 2017;9:114-31.

39. Paieri F, Tadini L, Manavski N, Kleine T, Ferrari R, Morandini P, Pesaresi $P$, Meurer J, Leister D. The DEAD-box RNA helicase RH50 is a 23S-4.5S rRNA maturation factor that functionally overlaps with the plastid signaling factor GUN1. Plant Physiol. 2018:176:634-48.

40. Tamburino R, Vitale M, Ruggiero A, Sassi M, Sannino L, Arena S, Costa A, Batelli G, Zambrano N, Scaloni A, Grillo S, Scotti N. Chloroplast proteome response to drought stress and recovery in tomato (Solanum lycopersicum L.). BMC Plant Biol. 2017;17:40.

41. Russell R. RNA misfolding and the action of chaperones. Front Biosci. 2008; 13:1-20.

42. Semrad K. Proteins with RNA chaperone activity: a world of diverse proteins with a common task-impediment of RNA misfolding. Biochem Res Int. 2011 10:1155-65. 
43. Bravo JPK, Borodavka A, Barth A, Calabrese AN, Mojzes PJ, Cockburn JB, Tuma R. Stability of local secondary structure determines selectivity of viral RNA chaperones. Nucleic Acid Res. 2018. https://doi.org/10.1093/nar/gky394.

44. Cordin O, Banroques J, Tanner NK, Linder P. The DEAD-box protein family of RNA helicases. Gene. 2006;367:17-37.

45. Melencion SMB, Chi YH, Pham TT, Paeng SK, Wi SD, et al. RNA chaperone function of a universal stress protein in Arabidopsis confers enhanced cold stress tolerance in plants. Int J Mol Sci. 2017;18:2546.

46. Mohr S, Stryker JM, Lambowitz AM. A DEAD-box protein functions as an ATP dependent RNA chaperone in group I intron splicing. Cell. 2002;109: 769-79.

47. Halls C, Mohr S, del Campo M, Yang Q, Jankowsky E, Lambowitz AM. Involvement of DEAD-box proteins in group I and group II intron splicing. Biochemical characterization of Mss116p, ATP hydrolysis-dependent and independent mechanisms, and general RNA chaperone activity. J Mol Biol. 2007;365:835-55.

48. Turk EM, Caprara MG. Splicing of yeast al5 $\beta$ group I intron requires SUV3 to recycle MRS1 via mitochondrial degradosome-promoted decay of excised intron ribonucleoprotein (RNP). J Biol Chem. 2010;285:8585-94.

49. Russell R, Jarmoskaite I, Lambowitz AM. Toward a molecular understanding of RNA remodeling by DEAD-box proteins. RNA Biol. 2013;10:44-55.

50. Jarmoskaite I, Bhaskaran H, Seifert S, Russell R. DEAD-box protein CYT-19 is activated by exposed helices in a group I intron RNA. Proc Natl Acad Sci U S A. 2014;22:2928-36.

51. Kim J, Le TNN, Kang H. Artificial targeting of a nucleus-encoded RNAbinding protein AtRZ1a to chloroplasts affects flowering and ABA response of Arabidopsis thaliana. J Plant Biol. 2017;60:278-84.

52. Lee K, Lee HJ, Kim DH, Jeon Y, Pai H-S, Kang H. A nuclear-encoded chloroplast protein harboring a single CRM domain plays an important role in the Arabidopsis growth and stress response. BMC Plant Biol. 2014;14:98.

53. Lee K, Han JH, Park YI, Colas d, Francs-Small C, Small I, Kang H. The mitochondrial pentatricopeptide repeat protein PPR19 is involved in the stabilization of $\mathrm{NADH}$ dehydrogenase 1 transcripts and is crucial for mitochondrial function and Arabidopsis thaliana development. New Phytol. 2017;215:202-16

54. Bechtold N, Pelletier G. In planta Agrobacterium-mediated transformation of adult Arabidopsis thaliana plants by vacuum infiltration. Methods Mol Biol. 1998:82:259-66.

Ready to submit your research? Choose BMC and benefit from:

- fast, convenient online submission

- thorough peer review by experienced researchers in your field

- rapid publication on acceptance

- support for research data, including large and complex data types

- gold Open Access which fosters wider collaboration and increased citations

- maximum visibility for your research: over $100 \mathrm{M}$ website views per year

At $\mathrm{BMC}$, research is always in progress.

Learn more biomedcentral.com/submissions 